\title{
A Conjugate Gradient Algorithm under Yuan-Wei-Lu Line Search Technique for Large-Scale Minimization Optimization Models
}

\author{
Xiangrong Li $\mathbb{D}^{1},{ }^{1}$ Songhua Wang, ${ }^{2}$ Zhongzhou Jin, ${ }^{3}$ and Hongtruong Pham ${ }^{4}$ \\ ${ }^{1}$ College of Mathematics and Information Science, Guangxi University, Nanning, Guangxi 530004, China \\ ${ }^{2}$ School of Mathematics and Statistics, Baise University, Baise, Guangxi 533000, China \\ ${ }^{3}$ Business School, Guangxi University, Nanning, Guangxi 530004, China \\ ${ }^{4}$ Thai Nguyen University of Economics and Business Administration, Thai Nguyen, Vietnam
}

Correspondence should be addressed to Xiangrong Li; xrli68@163.com

Received 17 October 2017; Accepted 27 November 2017; Published 15 January 2018

Academic Editor: Guillermo Cabrera-Guerrero

Copyright (C) 2018 Xiangrong Li et al. This is an open access article distributed under the Creative Commons Attribution License, which permits unrestricted use, distribution, and reproduction in any medium, provided the original work is properly cited.

\begin{abstract}
This paper gives a modified Hestenes and Stiefel (HS) conjugate gradient algorithm under the Yuan-Wei-Lu inexact line search technique for large-scale unconstrained optimization problems, where the proposed algorithm has the following properties: (1) the new search direction possesses not only a sufficient descent property but also a trust region feature; (2) the presented algorithm has global convergence for nonconvex functions; (3) the numerical experiment showed that the new algorithm is more effective than similar algorithms.
\end{abstract}

\section{Introduction}

Consider the minimization optimization models defined by

$$
\min \left\{f(x) \mid x \in \mathfrak{R}^{n}\right\}
$$

where the function $f: \mathfrak{R}^{n} \rightarrow \mathfrak{R}$ and $f \in C^{2}$. There exist many good algorithms for (1), such as the quasi-Newton methods [1] and the conjugate gradient methods [2-5], where the iterative formula of the conjugate gradient algorithm for (1) is designed by

$$
x_{k+1}=x_{k}+\alpha_{k} d_{k}, \quad k=0,1,2, \ldots,
$$

where $x_{k}$ is the $k$ th iterative point, $\alpha_{k}$ is the steplength, and $d_{k}$ is the so-called conjugate gradient search direction with

$$
d_{k+1}= \begin{cases}-g\left(x_{k+1}\right)+\beta_{k} d_{k}, & \text { if } k \geq 1, \\ -g\left(x_{k+1}\right), & \text { if } k=0,\end{cases}
$$

where $\beta_{k}$ is a scalar determined from different conjugate gradient formulas and the HS method [3] is one of the most well-known conjugate gradient methods, which is

$$
\beta_{k}^{\mathrm{HS}}=\frac{g_{k+1}^{T} y_{k}}{d_{k}^{T} y_{k}},
$$

where $y_{k}=g_{k+1}-g_{k}, g_{k+1}=\nabla f\left(x_{k+1}\right)$ and $g_{k}=$ $\nabla f\left(x_{k}\right)$. The HS method has good numerical results for (1); however, the convergent theory is not interesting especially for the nonconvex function. At present, there exist many good conjugate gradients (see [6-8], etc.). Yuan, Wei, and Lu [9] gave a modified weak Wolfe-Powell (we called it YWL) line search for steplength $\alpha_{k}$ designed by

$$
\begin{aligned}
& f\left(x_{k}+\alpha_{k} d_{k}\right) \\
& \quad \leq f_{k}+\delta \alpha_{k} g_{k}^{T} d_{k}+\alpha_{k} \min \left[-\delta_{1} g_{k}^{T} d_{k}, \delta \frac{\alpha_{k}}{2}\left\|d_{k}\right\|^{2}\right], \\
& g\left(x_{k}+\alpha_{k} d_{k}\right)^{T} d_{k} \\
& \quad \geq \sigma g_{k}^{T} d_{k}+\min \left[-\delta_{1} g_{k}^{T} d_{k}, \delta \alpha_{k}\left\|d_{k}\right\|^{2}\right],
\end{aligned}
$$


where $\delta \in(0,1 / 2), \delta_{1} \in(0, \delta), \sigma \in(\delta, 1)$, and $\|\cdot\|$ denotes the Euclidean norm. It is well known that there exist two open problems which are the global convergence of the normal BFGS method and the global convergence of the PRP method for nonconvex functions under the inexact line search technique, where the first problem is regarded as one of the most difficult one thousand mathematical problems of the 20th century [10]. Yuan et al. [9] partly solved these two open problems under the YWL technique, and the numerical performance shows that the YWL technique is more competitive than the normal weak Wolfe-Powell technique. Further study work can be found in their paper [11]. By (5), it is not difficult to see that the YWL conditions are equivalent to the weak Wolfe-Powell (WWP) conditions if $-\delta_{1} g_{k}^{T} d_{k}<\delta\left(\alpha_{k} / 2\right)\left\|d_{k}\right\|^{2}$ holds, which implies that the YWL technique includes the WWP technique in some sense. Motivated by the above observations, we will make a further study and propose a new algorithm for (1). The main features of this paper are as follows:

(i) A modified HS conjugate gradient formula is given, which has not only a sufficient descent property but also a trust region feature. (ii) The global convergence of the given HS conjugate gradient algorithm for nonconvex functions is established.

(iii) Numerical results show that the new HS conjugate gradient algorithm under the YWL line search technique is better than the normal weak Wolfe-Powell technique.

This paper is organized as follows. In Section 2, a modified HS conjugate gradient algorithm is introduced. The global convergence of the given algorithm for nonconvex functions is established in Section 3 and numerical results are reported in Section 4.

\section{Motivation and Algorithm}

The nonlinear conjugate gradient algorithm is simple and has low memory requirement properties and is very effective for large-scale optimization problems, where the HS method is one of the most effective methods. However, the normal HS method has good numerical performance but fails in the convergence of nonconvex functions under the inexact line search technique. In order to overcome this shortcoming, a modified HS formula is defined by

$$
d_{k+1}= \begin{cases}-g_{k+1}+\frac{g_{k+1}^{T} y_{k} d_{k}-d_{k}^{T} g_{k+1} y_{k}}{\psi_{1}\left\|d_{k}\right\|^{2}+2 \psi_{2}\left\|d_{k}\right\|\left\|y_{k}\right\|+\left\|g_{k}\right\|^{2}+\psi_{3}\left\|y_{k}\right\|^{2},} & \text { if } k \geq 1, \\ -g_{k+1}, & \text { if } k=0,\end{cases}
$$

where $y_{k}=g_{k+1}-g_{k}$ and $\psi_{1}, \psi_{2}$, and $\psi_{3}$ are positive constants. This formula is inspired by the idea of these two papers $[6,8]$. In recent years, lots of scholars like to study the three-term conjugate gradient formula because of its good properties [7]. In the next section, we will prove that the new formula possesses not only a sufficient descent property but also a trust region feature. The sufficient descent property is good for the convergence and the trust region makes the convergence easy to prove. Now, we give the steps of the proposed algorithm as follows.

Algorithm 1 (the modified three-term HS conjugate gradient algorithm (M-TT-HS-A)).

Step 1. $x_{1} \in \mathfrak{R}^{n}, \epsilon \in(0,1), \delta \in(0,1 / 2), \delta_{1} \in(0, \delta), \sigma \in$ $(\delta, 1), \psi_{1}>0, \psi_{2}>0, \psi_{3}>0$. Let $k=1$ and $d_{1}=-g\left(x_{1}\right)$.

Step 2. If $\left\|g_{k}\right\| \leq \epsilon$ holds, stop.

Step 3. Find $\alpha_{k}$ by the YWL line search satisfying (5) and (6).

Step 4. Let $x_{k+1}=x_{k}+\alpha_{k} d_{k}$.

Step 5. Compute the direction $d_{k+1}$ by (7).

Step 6. The algorithm stops if $\left\|g_{k+1}\right\| \leq \epsilon$.

Step 7. Let $k=k+1$ and go to Step 3 .

\section{Sufficient Descent Property, Trust Region Feature, and Global Convergence}

This section will prove some properties of Algorithm 1.

Lemma 2. The search direction $d_{k}$ is designed by (7); the following two relations hold:

$$
\begin{aligned}
g_{k}^{T} d_{k} & =-\left\|g_{k}\right\|^{2}, \\
\left\|d_{k}\right\| & \leq \psi^{*}\left\|g_{k}\right\|,
\end{aligned}
$$

where $\psi^{*}>0$ is a constant.

Proof. If $k=1$, it is easy to have (8) and (9). If $k \geq 1$, by formula (7), we have

$$
\begin{aligned}
& g_{k+1}^{T} d_{k+1}=g_{k+1}^{T}\left[-g_{k+1}\right. \\
& \left.+\frac{g_{k+1}^{T} y_{k} d_{k}-d_{k}^{T} g_{k+1} y_{k}}{\psi_{1}\left\|d_{k}\right\|^{2}+2 \psi_{2}\left\|d_{k}\right\|\left\|y_{k}\right\|+\left\|g_{k}\right\|^{2}+\psi_{3}\left\|y_{k}\right\|^{2}}\right] \\
& \quad=-\left\|g_{k+1}\right\|^{2} \\
& +\frac{g\left(x_{k+1}\right)^{T} y_{k} g_{k+1}^{T} d_{k}-d_{k}^{T} g\left(x_{k+1}\right) g_{k+1}^{T} y_{k}}{\psi_{1}\left\|d_{k}\right\|^{2}+2 \psi_{2}\left\|d_{k}\right\|\left\|y_{k}\right\|+\left\|g_{k}\right\|^{2}+\psi_{3}\left\|y_{k}\right\|^{2}}
\end{aligned}
$$




$$
\begin{aligned}
& =-\left\|g_{k+1}\right\|^{2}, \\
& \left\|d_{k+1}\right\|=\|-g\left(x_{k+1}\right) \\
& +\frac{g_{k+1}^{T} y_{k} d_{k}-d_{k}^{T} g_{k+1} y_{k}}{\psi_{1}\left\|d_{k}\right\|^{2}+2 \psi_{2}\left\|d_{k}\right\|\left\|y_{k}\right\|+\left\|g_{k}\right\|^{2}+\psi_{3}\left\|y_{k}\right\|^{2} \|} \\
& \leq\left\|g_{k+1}\right\| \\
& +\frac{\left\|g\left(x_{k+1}\right)\right\|\left\|y_{k}\right\|\left\|d_{k}\right\|+\left\|d_{k}\right\|\left\|g\left(x_{k+1}\right)\right\|\left\|y_{k}\right\|}{\psi_{1}\left\|d_{k}\right\|^{2}+2 \psi_{2}\left\|d_{k}\right\|\left\|y_{k}\right\|+\left\|g_{k}\right\|^{2}+\psi_{3}\left\|y_{k}\right\|^{2}} \\
& \leq\left\|g_{k+1}\right\|+\frac{2\left\|g\left(x_{k+1}\right)\right\|\left\|y_{k}\right\|\left\|d_{k}\right\|}{2 \psi_{2}\left\|d_{k}\right\|\left\|y_{k}\right\|} \leq\left(1+\frac{1}{\psi_{2}}\right) \\
& \cdot\left\|g_{k+1}\right\| .
\end{aligned}
$$

Then, (8) holds as well as (9) by letting $\psi^{*} \in\left[1+1 / \psi_{2},+\infty\right)$. This completes the proof.

Inequality (8) shows that the new formula has a sufficient descent property and inequality (9) proves that the new formula possesses a trust region feature. Both of these properties (8) and (9) are good theory characters and they play an important role in the global convergence of a conjugate gradient algorithm. The following global convergence theory will explain all this.

The following general assumptions are needed.

Assumption A. (i) The defined level set $L_{0}=\{x \mid f(x) \leq$ $\left.f\left(x_{1}\right)\right\}$ is bounded.

(ii) The objective function $f(x)$ is bounded below, twice continuously differentiable, and is Lipschitz continuous; namely, the following inequality is true:

$$
\|g(x)-g(y)\| \leq L\|x-y\|, \quad x, y \in \Re^{n},
$$

where $L>0$ is the Lipschitz constant.

By Lemma 2 and Assumption A, similar to [9], it is not difficult to show that the YWL line search technique is reasonable and Algorithm 1 is well defined. Here, we do not state it anymore. Now, we prove the global convergence of Algorithm 1 for nonconvex functions.

Theorem 3. Let Assumption A hold, and the iterate sequence $\left\{x_{k}, \alpha_{k}, d_{k}, g\left(x_{k}\right)\right\}$ is generated by M-TT-HS-A. Then, the relation

$$
\lim _{k \rightarrow \infty}\left\|g_{k}\right\|=0
$$

is true.
Proof. By (5), (8), and (9), we obtain

$$
\begin{aligned}
f\left(x_{k}+\alpha_{k} d_{k}\right) \leq & f\left(x_{k}\right)+\delta \alpha_{k} g_{k}^{T} d_{k} \\
& +\alpha_{k} \min \left[-\delta_{1} g_{k}^{T} d_{k}, \delta \frac{\alpha_{k}}{2}\left\|d_{k}\right\|^{2}\right] \\
\leq & f\left(x_{k}\right)+\delta \alpha_{k} g_{k}^{T} d_{k}-\alpha_{k} \delta_{1} g_{k}^{T} d_{k} \\
\leq & f\left(x_{k}\right)+\alpha_{k}\left(\delta-\delta_{1}\right) g_{k}^{T} d_{k} \\
\leq & f\left(x_{k}\right)-\alpha_{k}\left(\delta-\delta_{1}\right)\left\|g_{k}\right\|^{2} .
\end{aligned}
$$

Summing these inequalities for $k=0$ to $\infty$ and using Assumption A (ii) generate

$$
\sum_{k=0}^{\infty}\left(\gamma_{1}-\gamma\right) \alpha_{k}\left(\delta-\delta_{1}\right)\left\|g_{k}\right\|^{2} \leq f\left(x_{1}\right)-f_{\infty}<+\infty .
$$

Inequality (14) implies that

$$
\lim _{k \rightarrow \infty} \alpha_{k}\left\|g_{k}\right\|^{2}=0
$$

is true. By (6) and (8) again, we get

$$
\begin{aligned}
g\left(x_{k}+\alpha_{k} d_{k}\right)^{T} d_{k} \geq & \sigma g_{k}^{T} d_{k} \\
& +\min \left[-\delta_{1} g_{k}^{T} d_{k}, \delta \alpha_{k}\left\|d_{k}\right\|^{2}\right] \\
\geq & \sigma g_{k}^{T} d_{k} .
\end{aligned}
$$

Thus, the inequality

$$
\begin{aligned}
-(\sigma-1)\left\|g_{k}\right\|^{2} & \leq(\sigma-1) g_{k}^{T} d_{k} \\
& \leq\left[g\left(x_{k}+\alpha_{k} d_{k}\right)-g\left(x_{k}\right)\right]^{T} d_{k} \\
& \leq\left\|g\left(x_{k}+\alpha_{k} d_{k}\right)-g\left(x_{k}\right)\right\|\left\|d_{k}\right\| \\
& \leq \alpha_{k} L\left\|d_{k}\right\|^{2}
\end{aligned}
$$

holds, where the first inequality follows (8) and the last inequality follows (11). Then, we have

$$
\alpha_{k} \geq \frac{(1-\sigma)\left\|g_{k}\right\|^{2}}{L\left\|d_{k}\right\|^{2}} \geq \frac{(1-\sigma)\left\|g_{k}\right\|^{2}}{L\left(\psi^{*}\right)^{2}\left\|g_{k}\right\|^{2}}=\frac{(1-\sigma)}{L\left(\psi^{*}\right)^{2}} .
$$

By (15) and (18), we have

$$
\lim _{k \rightarrow \infty}\left\|g_{k}\right\|^{2}=0 .
$$

Therefore, we get (12) and the proof is complete.

\section{Numerical Results Performance}

This section will give numerical results of Algorithm 1 and the similar algorithms for comparing them. We will give another two algorithms for comparison; they are listed as follows. 
Algorithm 2 (the normal three-term formula [8] under the YWL technique).

Step 1. $x_{1} \in \mathfrak{R}^{n}, \epsilon \in(0,1), \delta \in(0,1 / 2), \delta_{1} \in(0, \delta), \sigma \in$ $(\delta, 1), \psi_{1}>0, \psi_{2}>0, \psi_{3}>0$. Let $k=1$ and $d_{1}=-g\left(x_{1}\right)$.

Step 2. If $\left\|g_{k}\right\| \leq \epsilon$ holds, stop.

Step 3. Find $\alpha_{k}$ by the YWL line search satisfying (5) and (6).

Step 4. Let $x_{k+1}=x_{k}+\alpha_{k} d_{k}$.

Step 5. Compute the direction $d_{k+1}$ by

$$
d_{k+1}= \begin{cases}-g_{k+1}+\frac{g_{k+1}^{T} y_{k} d_{k}-d_{k}^{T} g_{k+1} y_{k}}{\left\|g_{k}\right\|^{2},} & \text { if } k \geq 1, \\ -g_{k+1}, & \text { if } k=0 .\end{cases}
$$

Step 6. The algorithm stops if $\left\|g_{k+1}\right\| \leq \epsilon$.

Step 7. Let $k=k+1$ and go to Step 3 .

Algorithm 3 (the normal three-term formula [8] under the WWP technique).

Step 1. $x_{1} \in \mathfrak{R}^{n}, \epsilon \in(0,1), \delta \in(0,1 / 2), \sigma \in(\delta, 1)$. Let $k=1$ and $d_{1}=-g\left(x_{1}\right)$.

Step 2. If $\left\|g_{k}\right\| \leq \epsilon$ holds, stop.

Step 3. Find $\alpha_{k}$ by the WWP line search satisfying

$$
\begin{aligned}
f\left(x_{k}+\alpha_{k} d_{k}\right) & \leq f_{k}+\delta \alpha_{k} g_{k}^{T} d_{k}, \\
g\left(x_{k}+\alpha_{k} d_{k}\right)^{T} d_{k} & \geq \sigma g_{k}^{T} d_{k} .
\end{aligned}
$$

Step 4. Let $x_{k+1}=x_{k}+\alpha_{k} d_{k}$.

Step 5. Compute the direction $d_{k+1}$ by (20).

Step 6. The algorithm stops if $\left\|g_{k+1}\right\| \leq \epsilon$.

Step 7. Let $k=k+1$ and go to Step 3 .

4.1. Problems and Experiment. The following are some notes.

Test Problems. These problems and the related initial points are listed in Table 1; the detailed problems can be found in Andrei [12], and some papers also use these problems [13].

Experiments. Codes are run on Intel(R) Xeon(R) CPU, E5507 @2.27 GHz, and 6.00 GB memory and Windows 7 operation system and written by MATLAB R2009a.

Parameters. $\delta=0.1, \delta_{1}=0.05, \sigma=0.9$, and $\psi_{1}=\psi_{2}=\psi_{3}=$ 0.001 .

Dimension. Large-scale dimensions $n=3000,6000,12000$, and 30000 .

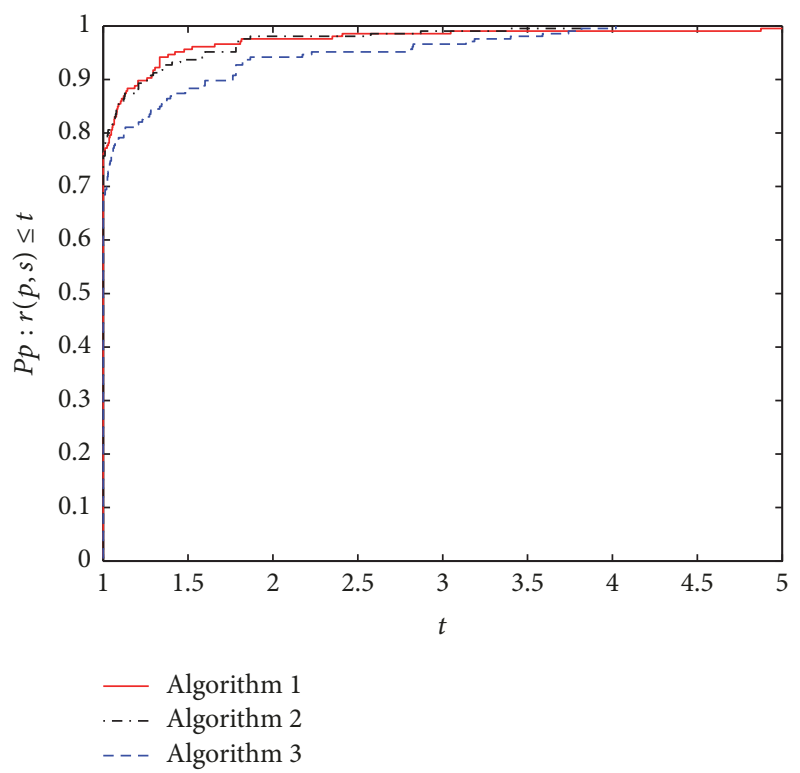

FIGURE 1: NI performance of these methods.

Stop Rules. Himmeblau stop rule: if $\left|f\left(x_{k}\right)\right|>1 e-5$, let stop1 $=\left|f\left(x_{k}\right)-f\left(x_{k+1}\right)\right| /\left|f\left(x_{k}\right)\right|$; otherwise, set stop1 $=$ $\left|f\left(x_{k}\right)-f\left(x_{k+1}\right)\right|$. If stop $1<1 e-5$ or $\left\|g\left(x_{k}\right)\right\|<1 e-6$ holds, the program stops.

Other Cases. The line search technique accepts $\alpha_{k}$ if the searching number is more than 6 and the algorithm will stop if the total iteration number is larger than 800 .

The numerical results are listed in Table 2, where

"Number" is the tested problems number;

"Dim." is the problems dimension;

"NI" is the total iteration number;

"CPU" is the system CPU time in seconds;

"NFG" is the total number of functions and gradients.

4.2. Results and Discussion. We use the tool of Dolan and Moré [14] to analyze the efficiency of the three given algorithms. Figures 1 and 2 show that the performance of Algorithm 1 is the best and that Algorithm 1 has the best robust property among those three methods and Algorithm 2 is better than Algorithm 3, which shows that the given formula (7) is competitive to the normal three-term conjugate gradient formula (20) and the YWL line search technique is more effective than the norm WWP technique, and all of these conclusions are coincident with the results of [9]. Algorithm 1 in Figure 3 is competitive to the other two algorithms and it has the best robust property. It is not difficult to see that Figure 3 shows that Algorithm 1 is not so good and we think the reason is formula (7) or the YWL technique since more information is needed and hence more $\mathrm{CPU}$ time is necessary. 
TABLE 1: Test problems.

\begin{tabular}{|c|c|c|}
\hline Number & Problem & $x_{0}$ \\
\hline (1) & Extended Freudenstein and Roth Function & {$[0.5,-2, \ldots, 0.5,-2]$} \\
\hline (2) & Extended Trigonometric Function & {$[0.2,0.2, \ldots, 0.2]$} \\
\hline (3) & Extended Rosenbrock Function & {$[-1.2,1,-1.2,1, \ldots,-1.2,1]$} \\
\hline (4) & Extended Beale Function & {$[1,0.8, \ldots, 1,0.8]$} \\
\hline (5) & Raydan 1 Function & {$[1,1, \ldots, 1]$} \\
\hline (6) & Raydan 2 Function & {$[1,1, \ldots, 1]$} \\
\hline (7) & Diagonal 1 Function & {$[1 / n, 1 / n, \ldots, 1 / n]$} \\
\hline (8) & Diagonal 3 Function & {$[1,1, \ldots, 1]$} \\
\hline (9) & Hager Function & {$[1,1, \ldots, 1]$} \\
\hline (10) & Generalized Tridiagonal 1 Function & {$[2,2, \ldots, 2]$} \\
\hline (11) & Extended Tridiagonal 1 Function & {$[2,2, \ldots, 2]$} \\
\hline (12) & Extended Three Exponential Terms Function & {$[0.1,0.1, \ldots, 0.1]$} \\
\hline (13) & Diagonal 4 Function & {$[1,1, \ldots, 1,1]$} \\
\hline (14) & Diagonal 5 Function & {$[1.1,1.1, \ldots, 1.1]$} \\
\hline (15) & Extended Himmelblau Function & {$[1,1, \ldots, 1]$} \\
\hline (16) & Generalized PSC1 Function & {$[3,0.1, \ldots, 3,0.1]$} \\
\hline (17) & Extended PSC1 Function & {$[3,0.1, \ldots, 3,0.1]$} \\
\hline (18) & Extended Block Diagonal BD1 Function & {$[0.1,0.1, \ldots, 0.1]$} \\
\hline (19) & Extended Maratos Function & {$[1.1,0.1, \ldots, 1.1,0.1]$} \\
\hline (20) & Extended Cliff Function & {$[0,-1, \ldots, 0,-1]$} \\
\hline (21) & Extended Wood Function & {$[-3,-1,-3,-1, \ldots,-3,-1]$} \\
\hline (22) & Extended Quadratic Penalty QP1 Function & {$[1,1, \ldots, 1]$} \\
\hline (23) & Extended Quadratic Penalty QP2 Function & {$[1,1, \ldots, 1]$} \\
\hline (24) & A Quadratic Function QF2 Function & {$[0.5,0.5, \ldots, 0.5]$} \\
\hline (25) & Extended EP1 Function & {$[1.5,1.5, \ldots, 1.5]$} \\
\hline$(26)$ & Extended Tridiagonal-2 Function & {$[1,1, \ldots, 1]$} \\
\hline (27) & BDQRTIC Function (CUTE) & {$[1,1, \ldots, 1]$} \\
\hline (28) & ARWHEAD Function (CUTE) & {$[1,1, \ldots, 1]$} \\
\hline (29) & NONDIA (Shanno-78) Function (CUTE) & {$[-1,-1, \ldots,-1]$} \\
\hline$(30)$ & DQDRTIC Function (CUTEr) & {$[3,3,3, \ldots, 3]$} \\
\hline (31) & EG2 Function (CUTE) & {$[1,1,1, \ldots, 1]$} \\
\hline (32) & DIXMAANA Function (CUTE) & {$[2,2,2, \ldots, 2]$} \\
\hline (33) & DIXMAANB Function (CUTE) & {$[2,2,2, \ldots, 2]$} \\
\hline (34) & DIXMAANC Function (CUTE) & {$[2,2,2, \ldots, 2]$} \\
\hline (35) & Partial Perturbed Quadratic Function & {$[0.5,0.5, \ldots, 0.5]$} \\
\hline (36) & Broyden Tridiagonal Function & {$[-1,-1, \ldots,-1]$} \\
\hline (37) & EDENSCH Function (CUTE) & {$[0,0, \ldots, 0]$} \\
\hline (38) & LIARWHD Function (CUTEr) & {$[4,4, \ldots, 4]$} \\
\hline$(39)$ & DIAGONAL 6 Function & {$[1,1, \ldots, 1]$} \\
\hline$(40)$ & DIXON3DQ Function (CUTE) & {$[-1,-1, \ldots,-1]$} \\
\hline (41) & DIXMAANF Function (CUTE) & {$[2,2,2, \ldots, 2]$} \\
\hline$(42)$ & DIXMAANG Function (CUTE) & {$[2,2,2, \ldots, 2]$} \\
\hline (43) & DIXMAANH Function (CUTE) & {$[2,2,2, \ldots, 2]$} \\
\hline$(44)$ & DIXMAANI Function (CUTE) & {$[2,2,2, \ldots, 2]$} \\
\hline$(45)$ & DIXMAANJ Function (CUTE) & {$[2,2,2, \ldots, 2]$} \\
\hline$(46)$ & DIXMAANK Function (CUTE) & {$[2,2,2, \ldots, 2]$} \\
\hline$(47)$ & DIXMAANL Function (CUTE) & {$[2,2,2, \ldots, 2]$} \\
\hline$(48)$ & DIXMAAND Function (CUTE) & {$[2,2,2, \ldots, 2]$} \\
\hline (49) & ENGVAL1 Function (CUTE) & {$[2,2,2, \ldots, 2]$} \\
\hline$(50)$ & Extended DENSCHNB Function (CUTE) & {$[1,1, \ldots, 1]$} \\
\hline (51) & SINQUAD Function (CUTE) & {$[0.1,0.1, \ldots, 0.1]$} \\
\hline$(52)$ & Partial Perturbed Quadratic PPQ2 Function & {$[0.5,0.5, \ldots, 0.5]$} \\
\hline
\end{tabular}


TABLE 2: Numerical results.

\begin{tabular}{|c|c|c|c|c|c|c|c|c|c|c|}
\hline \multirow{2}{*}{ Number } & \multirow{2}{*}{ Dim. } & \multicolumn{3}{|c|}{ Algorithm 1} & \multicolumn{3}{|c|}{ Algorithm 2} & \multicolumn{3}{|c|}{ Algorithm 3} \\
\hline & & NI & NFG & $\mathrm{CPU}$ & $\mathrm{NI}$ & NFG & $\mathrm{CPU}$ & NI & NFG & $\mathrm{CPU}$ \\
\hline \multirow{4}{*}{ (1) } & 3000 & 15 & 38 & 0.093601 & 20 & 56 & 0.140401 & 20 & 56 & 0.124801 \\
\hline & 6000 & 24 & 66 & 0.358802 & 21 & 59 & 0.280802 & 21 & 59 & 0.249602 \\
\hline & 12000 & 22 & 59 & 0.577204 & 17 & 46 & 0.405603 & 17 & 46 & 0.405603 \\
\hline & 30000 & 22 & 59 & 1.357209 & 17 & 46 & 0.998406 & 17 & 46 & 1.029607 \\
\hline \multirow{4}{*}{ (2) } & 3000 & 74 & 163 & 0.327602 & 74 & 163 & 0.343202 & 83 & 181 & 0.358802 \\
\hline & 6000 & 81 & 176 & 0.702005 & 81 & 176 & 0.686404 & 81 & 176 & 0.655204 \\
\hline & 12000 & 83 & 182 & 1.388409 & 83 & 182 & 1.341609 & 83 & 182 & 1.357209 \\
\hline & 30000 & 88 & 194 & 3.572423 & 88 & 194 & 3.588023 & 89 & 196 & 3.478822 \\
\hline \multirow{4}{*}{ (3) } & 3000 & 88 & 262 & 0.124801 & 99 & 283 & 0.124801 & 73 & 216 & 0.093601 \\
\hline & 6000 & 83 & 277 & 0.234002 & 112 & 329 & 0.265202 & 112 & 329 & 0.249602 \\
\hline & 12000 & 78 & 272 & 0.390002 & 16 & 66 & 0.078001 & 16 & 66 & 0.093601 \\
\hline & 30000 & 49 & 119 & 0.483603 & 46 & 182 & 0.561604 & 46 & 182 & 0.468003 \\
\hline \multirow{4}{*}{ (4) } & 3000 & 32 & 87 & 0.109201 & 21 & 57 & 0.093601 & 21 & 57 & 0.0624 \\
\hline & 6000 & 24 & 65 & 0.171601 & 27 & 74 & 0.187201 & 27 & 74 & 0.187201 \\
\hline & 12000 & 23 & 62 & 0.312002 & 23 & 63 & 0.265202 & 26 & 69 & 0.343202 \\
\hline & 30000 & 24 & 67 & 0.748805 & 29 & 78 & 0.858005 & 25 & 66 & 0.717605 \\
\hline \multirow{4}{*}{ (5) } & 3000 & 23 & 51 & 0.0624 & 23 & 51 & 0.0312 & 23 & 51 & 0.0624 \\
\hline & 6000 & 23 & 51 & 0.093601 & 23 & 51 & 0.093601 & 23 & 51 & 0.0624 \\
\hline & 12000 & 23 & 51 & 0.171601 & 23 & 51 & 0.156001 & 23 & 51 & 0.124801 \\
\hline & 30000 & 23 & 51 & 0.390002 & 23 & 51 & 0.358802 & 23 & 51 & 0.312002 \\
\hline \multirow{4}{*}{ (6) } & 3000 & 12 & 26 & 0.0156 & 12 & 26 & 0.0156 & 12 & 26 & 0.0156 \\
\hline & 6000 & 12 & 26 & 0.0468 & 12 & 26 & 0.0468 & 12 & 26 & 0.0312 \\
\hline & 12000 & 12 & 26 & 0.093601 & 12 & 26 & 0.0624 & 12 & 26 & 0.078 \\
\hline & 30000 & 12 & 26 & 0.171601 & 12 & 26 & 0.171601 & 12 & 26 & 0.156001 \\
\hline \multirow{4}{*}{ (7) } & 3000 & 2 & 9 & 0.0312 & 2 & 9 & 0.0312 & 2 & 9 & 0.0312 \\
\hline & 6000 & 2 & 9 & 0 & 2 & 9 & 0 & 2 & 9 & 0 \\
\hline & 12000 & 2 & 9 & 0 & 2 & 9 & 0.0312 & 2 & 9 & 0.0312 \\
\hline & 30000 & 2 & 9 & 0.0312 & 2 & 9 & 0.0468 & 2 & 9 & 0.0468 \\
\hline \multirow{4}{*}{ (8) } & 3000 & 17 & 36 & 0.0624 & 17 & 36 & 0.0468 & 17 & 36 & 0.0624 \\
\hline & 6000 & 19 & 40 & 0.093601 & 19 & 40 & 0.109201 & 19 & 40 & 0.078 \\
\hline & 12000 & 19 & 40 & 0.156001 & 19 & 40 & 0.156001 & 19 & 40 & 0.171601 \\
\hline & 30000 & 19 & 40 & 0.405603 & 19 & 40 & 0.405603 & 19 & 40 & 0.374402 \\
\hline \multirow{4}{*}{ (9) } & 3000 & 24 & 113 & 0.078001 & 24 & 113 & 0.078 & 24 & 113 & 0.078 \\
\hline & 6000 & 22 & 52 & 0.093601 & 21 & 50 & 0.109201 & 22 & 52 & 0.109201 \\
\hline & 12000 & 2 & 9 & 0.0156 & 2 & 9 & 0.0156 & 2 & 9 & 0.0312 \\
\hline & 30000 & 2 & 9 & 0.0624 & 2 & 9 & 0.0468 & 2 & 9 & 0.078 \\
\hline \multirow{4}{*}{ (10) } & 3000 & 6 & 15 & 0.655204 & 6 & 15 & 0.592804 & 6 & 15 & 0.592804 \\
\hline & 6000 & 6 & 15 & 2.043613 & 6 & 15 & 1.981213 & 6 & 15 & 1.996813 \\
\hline & 12000 & 6 & 15 & 7.207246 & 6 & 15 & 7.503648 & 6 & 15 & 7.394447 \\
\hline & 30000 & 3 & 8 & 21.590538 & 3 & 8 & 21.528138 & 3 & 8 & 21.481338 \\
\hline \multirow{4}{*}{ (11) } & 3000 & 34 & 81 & 1.762811 & 35 & 86 & 1.653611 & 35 & 86 & 1.63801 \\
\hline & 6000 & 47 & 114 & 6.520842 & 34 & 77 & 4.383628 & 36 & 88 & 4.74243 \\
\hline & 12000 & 36 & 88 & 14.820095 & 37 & 93 & 15.241298 & 37 & 93 & 15.100897 \\
\hline & 30000 & 43 & 102 & 92.492993 & 39 & 100 & 86.050152 & 39 & 100 & 85.83175 \\
\hline \multirow{4}{*}{ (12) } & 3000 & 14 & 30 & 0.0468 & 14 & 30 & 0.0624 & 14 & 30 & 0.0468 \\
\hline & 6000 & 21 & 44 & 0.093601 & 21 & 44 & 0.109201 & 21 & 44 & 0.078001 \\
\hline & 12000 & 24 & 50 & 0.202801 & 24 & 50 & 0.218401 & 24 & 50 & 0.218401 \\
\hline & 30000 & 24 & 50 & 0.592804 & 24 & 50 & 0.483603 & 24 & 50 & 0.468003 \\
\hline & 3000 & 4 & 13 & 0.0312 & 3 & 10 & 0.0156 & 3 & 10 & 0 \\
\hline (13) & 6000 & 4 & 13 & 0 & 3 & 10 & 0 & 3 & 10 & 0 \\
\hline & 12000 & 4 & 13 & 0.0312 & 3 & 10 & 0.0312 & 3 & 10 & 0 \\
\hline & 30000 & 4 & 13 & 0.0468 & 3 & 10 & 0.0312 & 3 & 10 & 0.0312 \\
\hline
\end{tabular}


TABLe 2: Continued.

\begin{tabular}{|c|c|c|c|c|c|c|c|c|c|c|}
\hline \multirow{2}{*}{ Number } & \multirow{2}{*}{ Dim. } & \multicolumn{3}{|c|}{ Algorithm 1} & \multicolumn{3}{|c|}{ Algorithm 2} & \multicolumn{3}{|c|}{ Algorithm 3} \\
\hline & & $\mathrm{NI}$ & NFG & $\mathrm{CPU}$ & NI & NFG & $\mathrm{CPU}$ & NI & NFG & $\mathrm{CPU}$ \\
\hline \multirow{4}{*}{ (14) } & 3000 & 3 & 9 & 0.0312 & 3 & 9 & 0.0312 & 3 & 9 & 0 \\
\hline & 6000 & 3 & 9 & 0.0312 & 3 & 9 & 0.0312 & 3 & 9 & 0.0312 \\
\hline & 12000 & 3 & 9 & 0.0624 & 3 & 9 & 0.0624 & 3 & 9 & 0.0312 \\
\hline & 30000 & 3 & 9 & 0.078001 & 3 & 9 & 0.078 & 3 & 9 & 0.078001 \\
\hline \multirow{4}{*}{ (15) } & 3000 & 9 & 48 & 0.0468 & 9 & 48 & 0.0312 & 9 & 48 & 0.0312 \\
\hline & 6000 & 128 & 292 & 0.312002 & 42 & 108 & 0.093601 & 42 & 108 & 0.078 \\
\hline & 12000 & 24 & 114 & 0.140401 & 29 & 139 & 0.156001 & 29 & 139 & 0.156001 \\
\hline & 30000 & 13 & 39 & 0.156001 & 16 & 45 & 0.171601 & 16 & 45 & 0.156001 \\
\hline \multirow{4}{*}{ (16) } & 3000 & 33 & 76 & 0.109201 & 33 & 76 & 0.093601 & 42 & 90 & 0.093601 \\
\hline & 6000 & 32 & 73 & 0.187201 & 32 & 73 & 0.156001 & 41 & 88 & 0.187201 \\
\hline & 12000 & 32 & 73 & 0.312002 & 32 & 73 & 0.312002 & 43 & 95 & 0.390003 \\
\hline & 30000 & 33 & 78 & 0.811205 & 33 & 78 & 0.780005 & 37 & 86 & 0.889206 \\
\hline \multirow{4}{*}{ (17) } & 3000 & 9 & 44 & 0.124801 & 9 & 44 & 0.156001 & 9 & 43 & 0.124801 \\
\hline & 6000 & 9 & 44 & 0.280802 & 9 & 44 & 0.265202 & 9 & 44 & 0.249602 \\
\hline & 12000 & 9 & 44 & 0.499203 & 9 & 44 & 0.514803 & 9 & 44 & 0.514803 \\
\hline & 30000 & 9 & 44 & 1.279208 & 9 & 44 & 1.232408 & 9 & 44 & 1.232408 \\
\hline \multirow{4}{*}{ (18) } & 3000 & 11 & 88 & 0.0624 & 11 & 87 & 0.0312 & 11 & 86 & 0.0312 \\
\hline & 6000 & 11 & 88 & 0.093601 & 11 & 83 & 0.093601 & 11 & 83 & 0.093601 \\
\hline & 12000 & 47 & 207 & 0.436803 & 20 & 115 & 0.202801 & 20 & 115 & 0.187201 \\
\hline & 30000 & 39 & 158 & 0.873606 & 89 & 248 & 1.63801 & 31 & 134 & 0.733205 \\
\hline \multirow{4}{*}{ (19) } & 3000 & 28 & 56 & 0.0312 & 28 & 56 & 0.0468 & 28 & 56 & 0.0312 \\
\hline & 6000 & 38 & 76 & 0.078 & 38 & 76 & 0.093601 & 38 & 76 & 0.0624 \\
\hline & 12000 & 159 & 504 & 0.717605 & 170 & 594 & 0.733205 & 66 & 162 & 0.249602 \\
\hline & 30000 & 7 & 16 & 0.078 & 7 & 16 & 0.0468 & 7 & 16 & 0.078 \\
\hline \multirow{4}{*}{ (20) } & 3000 & 97 & 212 & 0.234002 & 97 & 212 & 0.218401 & 97 & 212 & 0.202801 \\
\hline & 6000 & 106 & 230 & 0.452403 & 106 & 230 & 0.421203 & 106 & 230 & 0.390003 \\
\hline & 12000 & 93 & 206 & 0.733205 & 93 & 206 & 0.702005 & 93 & 206 & 0.686404 \\
\hline & 30000 & 87 & 194 & 1.700411 & 87 & 194 & 1.54441 & 87 & 194 & 1.52881 \\
\hline \multirow{4}{*}{ (21) } & 3000 & 36 & 90 & 0.0468 & 36 & 90 & 0.0468 & 36 & 90 & 0.078 \\
\hline & 6000 & 37 & 94 & 0.124801 & 37 & 94 & 0.093601 & 37 & 94 & 0.093601 \\
\hline & 12000 & 34 & 88 & 0.202801 & 33 & 86 & 0.156001 & 33 & 86 & 0.156001 \\
\hline & 30000 & 33 & 83 & 0.436803 & 36 & 92 & 0.405603 & 36 & 92 & 0.421203 \\
\hline \multirow{4}{*}{ (22) } & 3000 & 42 & 92 & 0.0624 & 42 & 92 & 0.078 & 42 & 92 & 0.078 \\
\hline & 6000 & 41 & 90 & 0.109201 & 41 & 90 & 0.124801 & 41 & 90 & 0.093601 \\
\hline & 12000 & 41 & 90 & 0.234002 & 41 & 90 & 0.187201 & 41 & 90 & 0.202801 \\
\hline & 30000 & 45 & 98 & 0.514803 & 45 & 98 & 0.499203 & 45 & 98 & 0.483603 \\
\hline \multirow{4}{*}{ (23) } & 3000 & 42 & 88 & 0.109201 & 42 & 88 & 0.078001 & 42 & 88 & 0.078 \\
\hline & 6000 & 71 & 146 & 0.296402 & 71 & 146 & 0.296402 & 71 & 146 & 0.280802 \\
\hline & 12000 & 37 & 80 & 0.312002 & 37 & 80 & 0.265202 & 37 & 80 & 0.280802 \\
\hline & 30000 & 52 & 110 & 1.029607 & 52 & 110 & 0.904806 & 52 & 110 & 0.920406 \\
\hline \multirow{4}{*}{ (24) } & 3000 & 3 & 7 & 0 & 3 & 7 & 0 & 3 & 7 & 0 \\
\hline & 6000 & 3 & 7 & 0.0312 & 3 & 7 & 0.0312 & 3 & 7 & 0 \\
\hline & 12000 & 2 & 5 & 0 & 2 & 5 & 0 & 2 & 5 & 0 \\
\hline & 30000 & 2 & 5 & 0.0312 & 2 & 5 & 0.0312 & 2 & 5 & 0.0624 \\
\hline \multirow{4}{*}{ (25) } & 3000 & 4 & 8 & 0 & 4 & 8 & 0 & 4 & 8 & 0 \\
\hline & 6000 & 5 & 10 & 0.0156 & 5 & 10 & 0.0156 & 5 & 10 & 0.0312 \\
\hline & 12000 & 7 & 14 & 0.0312 & 7 & 14 & 0.0312 & 7 & 14 & 0.0624 \\
\hline & 30000 & 10 & 20 & 0.140401 & 10 & 20 & 0.124801 & 10 & 20 & 0.124801 \\
\hline & 3000 & 12 & 24 & 0.0312 & 12 & 24 & 0.0468 & 12 & 24 & 0.0468 \\
\hline (26) & 6000 & 16 & 32 & 0.078001 & 16 & 32 & 0.0468 & 16 & 32 & 0.0468 \\
\hline & 12000 & 21 & 42 & 0.124801 & 21 & 42 & 0.109201 & 21 & 42 & 0.109201 \\
\hline & 30000 & 30 & 62 & 0.421203 & 30 & 62 & 0.405603 & 31 & 62 & 0.405603 \\
\hline
\end{tabular}


TABLe 2: Continued.

\begin{tabular}{|c|c|c|c|c|c|c|c|c|c|c|}
\hline \multirow{2}{*}{ Number } & \multirow{2}{*}{ Dim. } & \multicolumn{3}{|c|}{ Algorithm 1} & \multicolumn{3}{|c|}{ Algorithm 2} & \multicolumn{3}{|c|}{ Algorithm 3} \\
\hline & & NI & NFG & CPU & NI & NFG & CPU & NI & NFG & CPU \\
\hline \multirow{4}{*}{ (27) } & 3000 & 18 & 57 & 1.341609 & 14 & 48 & 1.029607 & 14 & 48 & 0.998406 \\
\hline & 6000 & 5 & 16 & 1.060807 & 8 & 28 & 1.778411 & 8 & 28 & 1.778411 \\
\hline & 12000 & 5 & 16 & 3.494422 & 8 & 28 & 6.084039 & 8 & 28 & 6.068439 \\
\hline & 30000 & 5 & 16 & 21.138135 & 17 & 55 & 77.9693 & 17 & 55 & 77.9537 \\
\hline \multirow{4}{*}{$(28)$} & 3000 & 9 & 23 & 0.0312 & 9 & 26 & 0.0312 & 9 & 26 & 0.0156 \\
\hline & 6000 & 12 & 30 & 0.0312 & 12 & 30 & 0.0468 & 12 & 30 & 0.0312 \\
\hline & 12000 & 10 & 25 & 0.078 & 10 & 28 & 0.078001 & 10 & 28 & 0.0468 \\
\hline & 30000 & 12 & 29 & 0.140401 & 11 & 30 & 0.109201 & 11 & 30 & 0.124801 \\
\hline \multirow{4}{*}{ (29) } & 3000 & 26 & 52 & 0.0468 & 26 & 52 & 0.0312 & 26 & 52 & 0.0312 \\
\hline & 6000 & 27 & 54 & 0.078001 & 27 & 54 & 0.0468 & 27 & 54 & 0.0468 \\
\hline & 12000 & 29 & 58 & 0.124801 & 29 & 58 & 0.109201 & 29 & 58 & 0.093601 \\
\hline & 30000 & 23 & 46 & 0.249602 & 23 & 46 & 0.171601 & 23 & 46 & 0.202801 \\
\hline \multirow{4}{*}{ (30) } & 3000 & 49 & 119 & 0.0624 & 27 & 68 & 0.0624 & 27 & 68 & 0.0468 \\
\hline & 6000 & 37 & 92 & 0.109201 & 26 & 65 & 0.0468 & 26 & 65 & 0.078 \\
\hline & 12000 & 29 & 75 & 0.124801 & 28 & 69 & 0.124801 & 28 & 69 & 0.093601 \\
\hline & 30000 & 32 & 77 & 0.296402 & 27 & 68 & 0.234002 & 27 & 68 & 0.280802 \\
\hline \multirow{4}{*}{ (31) } & 3000 & 4 & 21 & 0 & 4 & 21 & 0 & 4 & 21 & 0 \\
\hline & 6000 & 4 & 21 & 0.0312 & 4 & 21 & 0.0156 & 4 & 21 & 0.0312 \\
\hline & 12000 & 4 & 21 & 0.0312 & 4 & 21 & 0.0312 & 4 & 21 & 0.0468 \\
\hline & 30000 & 4 & 21 & 0.093601 & 4 & 21 & 0.109201 & 4 & 21 & 0.109201 \\
\hline \multirow{4}{*}{ (32) } & 3000 & 22 & 48 & 0.296402 & 22 & 48 & 0.296402 & 22 & 48 & 0.265202 \\
\hline & 6000 & 23 & 50 & 0.577204 & 23 & 50 & 0.561604 & 23 & 50 & 0.608404 \\
\hline & 12000 & 24 & 52 & 1.201208 & 24 & 52 & 1.185608 & 24 & 52 & 1.185608 \\
\hline & 30000 & 25 & 54 & 3.026419 & 25 & 54 & 3.04202 & 25 & 54 & 3.010819 \\
\hline \multirow{4}{*}{ (33) } & 3000 & 38 & 80 & 0.483603 & 38 & 80 & 0.483603 & 36 & 76 & 0.436803 \\
\hline & 6000 & 39 & 82 & 0.951606 & 39 & 82 & 0.967206 & 37 & 78 & 0.889206 \\
\hline & 12000 & 41 & 86 & 1.950013 & 41 & 86 & 1.965613 & 38 & 80 & 1.856412 \\
\hline & 30000 & 42 & 88 & 5.054432 & 42 & 88 & 5.070032 & 39 & 82 & 4.69563 \\
\hline \multirow{4}{*}{$(34)$} & 3000 & 66 & 136 & 0.842405 & 66 & 136 & 0.795605 & 66 & 136 & 0.826805 \\
\hline & 6000 & 69 & 142 & 1.669211 & 69 & 142 & 1.669211 & 69 & 142 & 1.63801 \\
\hline & 12000 & 72 & 148 & 3.478822 & 72 & 148 & 3.447622 & 72 & 148 & 3.478822 \\
\hline & 30000 & 75 & 154 & 9.001258 & 75 & 154 & 8.970057 & 75 & 154 & 8.876457 \\
\hline \multirow{4}{*}{ (35) } & 3000 & 85 & 175 & 26.036567 & 86 & 177 & 26.364169 & 86 & 177 & 26.379769 \\
\hline & 6000 & 45 & 110 & 57.79837 & 84 & 180 & 103.631464 & 84 & 180 & 103.693865 \\
\hline & 12000 & 58 & 146 & 296.932303 & 35 & 98 & 186.514796 & 35 & 98 & 186.421195 \\
\hline & 30000 & 75 & 201 & 2473.645457 & 96 & 250 & 3169.191515 & 96 & 250 & 3169.331916 \\
\hline \multirow{4}{*}{ (36) } & 3000 & 33 & 76 & 1.279208 & 48 & 106 & 1.794012 & 49 & 105 & 1.809612 \\
\hline & 6000 & 47 & 106 & 7.020045 & 50 & 112 & 7.254046 & 50 & 112 & 7.254047 \\
\hline & 12000 & 49 & 101 & 26.410969 & 50 & 103 & 26.785372 & 50 & 103 & 26.769772 \\
\hline & 30000 & 40 & 95 & 154.050987 & 41 & 97 & 155.096194 & 41 & 97 & 154.940193 \\
\hline \multirow{4}{*}{ (37) } & 3000 & 23 & 48 & 0.124801 & 23 & 48 & 0.124801 & 23 & 48 & 0.124801 \\
\hline & 6000 & 23 & 48 & 0.265202 & 23 & 48 & 0.265202 & 23 & 48 & 0.265202 \\
\hline & 12000 & 23 & 48 & 0.499203 & 23 & 48 & 0.499203 & 23 & 48 & 0.483603 \\
\hline & 30000 & 23 & 48 & 1.232408 & 23 & 48 & 1.216808 & 23 & 48 & 1.170007 \\
\hline \multirow{4}{*}{ (38) } & 3000 & 12 & 36 & 0.0468 & 19 & 59 & 0.0468 & 19 & 59 & 0.0624 \\
\hline & 6000 & 47 & 150 & 0.156001 & 26 & 76 & 0.093601 & 26 & 76 & 0.0624 \\
\hline & 12000 & 68 & 178 & 0.374402 & 13 & 41 & 0.0624 & 13 & 41 & 0.093601 \\
\hline & 30000 & 11 & 30 & 0.140401 & 42 & 99 & 0.468003 & 42 & 99 & 0.452403 \\
\hline & 3000 & 21 & 44 & 0.483603 & 21 & 44 & 0.514803 & 21 & 44 & 0.483603 \\
\hline (39) & 6000 & 22 & 46 & 1.950013 & 22 & 46 & 1.934412 & 22 & 46 & 1.887612 \\
\hline ) & 12000 & 23 & 48 & 7.441248 & 23 & 48 & 7.441248 & 23 & 48 & 7.425648 \\
\hline & 30000 & 24 & 50 & 51.979533 & 24 & 50 & 51.121528 & 24 & 50 & 51.027927 \\
\hline
\end{tabular}


TABLE 2: Continued.

\begin{tabular}{|c|c|c|c|c|c|c|c|c|c|c|}
\hline \multirow{2}{*}{ Number } & \multirow{2}{*}{ Dim. } & \multicolumn{3}{|c|}{ Algorithm 1} & \multicolumn{3}{|c|}{ Algorithm 2} & \multicolumn{3}{|c|}{ Algorithm 3} \\
\hline & & NI & NFG & CPU & NI & NFG & $\mathrm{CPU}$ & $\mathrm{NI}$ & NFG & $\mathrm{CPU}$ \\
\hline \multirow{4}{*}{$(40)$} & 3000 & 449 & 906 & 0.468003 & 800 & 1619 & 0.826805 & 800 & 1615 & 0.780005 \\
\hline & 6000 & 449 & 906 & 0.748805 & 800 & 1619 & 1.294808 & 800 & 1615 & 1.279208 \\
\hline & 12000 & 449 & 906 & 1.263608 & 800 & 1619 & 2.371215 & 800 & 1615 & 2.308815 \\
\hline & 30000 & 449 & 906 & 3.07322 & 800 & 1619 & 5.288434 & 800 & 1615 & 5.163633 \\
\hline \multirow{4}{*}{ (41) } & 3000 & 93 & 192 & 1.170007 & 94 & 194 & 1.201208 & 164 & 339 & 2.028013 \\
\hline & 6000 & 73 & 152 & 1.825212 & 73 & 152 & 1.794012 & 204 & 419 & 5.038832 \\
\hline & 12000 & 80 & 166 & 3.931225 & 80 & 166 & 3.915625 & 251 & 513 & 12.246079 \\
\hline & 30000 & 90 & 186 & 11.029271 & 90 & 186 & 10.93567 & 323 & 657 & 39.07825 \\
\hline \multirow{4}{*}{$(42)$} & 3000 & 154 & 318 & 1.996813 & 150 & 310 & 1.887612 & 118 & 242 & 1.51321 \\
\hline & 6000 & 151 & 308 & 3.775224 & 151 & 323 & 3.822024 & 134 & 274 & 3.307221 \\
\hline & 12000 & 188 & 386 & 9.40686 & 202 & 414 & 9.937264 & 156 & 318 & 7.675249 \\
\hline & 30000 & 215 & 453 & 26.972573 & 239 & 492 & 29.390588 & 198 & 402 & 24.164555 \\
\hline \multirow{4}{*}{ (43) } & 3000 & 96 & 205 & 1.248008 & 71 & 158 & 0.889206 & 65 & 150 & 0.826805 \\
\hline & 6000 & 65 & 151 & 1.669211 & 58 & 129 & 1.48201 & 81 & 172 & 1.996813 \\
\hline & 12000 & 81 & 177 & 4.102826 & 85 & 185 & 4.212027 & 100 & 210 & 4.929632 \\
\hline & 30000 & 89 & 193 & 11.169672 & 90 & 195 & 11.122871 & 91 & 205 & 11.310072 \\
\hline \multirow{4}{*}{ (44) } & 3000 & 142 & 294 & 1.856412 & 137 & 284 & 1.731611 & 128 & 267 & 1.63801 \\
\hline & 6000 & 159 & 328 & 4.024826 & 153 & 316 & 3.837625 & 162 & 339 & 4.056026 \\
\hline & 12000 & 178 & 366 & 9.001258 & 170 & 350 & 8.455254 & 254 & 531 & 12.682881 \\
\hline & 30000 & 212 & 434 & 26.379769 & 199 & 408 & 24.554557 & 800 & 1612 & 99.060635 \\
\hline \multirow{4}{*}{$(45)$} & 3000 & 93 & 192 & 1.170007 & 94 & 194 & 1.185608 & 164 & 339 & 2.074813 \\
\hline & 6000 & 73 & 152 & 1.840812 & 73 & 152 & 1.825212 & 206 & 423 & 5.101233 \\
\hline & 12000 & 80 & 166 & 3.962425 & 80 & 166 & 3.900025 & 255 & 521 & 12.44888 \\
\hline & 30000 & 90 & 186 & 11.013671 & 89 & 184 & 10.85767 & 333 & 677 & 40.404259 \\
\hline \multirow{4}{*}{ (46) } & 3000 & 72 & 163 & 0.951606 & 73 & 176 & 0.982806 & 99 & 210 & 1.279208 \\
\hline & 6000 & 119 & 250 & 2.979619 & 115 & 242 & 2.839218 & 123 & 258 & 3.026419 \\
\hline & 12000 & 138 & 288 & 6.848444 & 147 & 306 & 7.238446 & 137 & 286 & 6.739243 \\
\hline & 30000 & 174 & 360 & 21.574938 & 172 & 356 & 21.091335 & 170 & 352 & 20.794933 \\
\hline \multirow{4}{*}{$(47)$} & 3000 & 302 & 613 & 3.946825 & 283 & 575 & 3.634823 & 800 & 1606 & 10.654868 \\
\hline & 6000 & 398 & 805 & 10.124465 & 368 & 745 & 9.31326 & 800 & 1606 & 20.950934 \\
\hline & 12000 & 359 & 727 & 18.189717 & 402 & 813 & 20.139729 & 800 & 1606 & 41.621067 \\
\hline & 30000 & 437 & 883 & 54.912352 & 437 & 883 & 54.366348 & 800 & 1606 & 103.584664 \\
\hline \multirow{4}{*}{ (48) } & 3000 & 26 & 58 & 0.343202 & 26 & 58 & 0.327602 & 27 & 60 & 0.327602 \\
\hline & 6000 & 27 & 60 & 0.686404 & 27 & 60 & 0.670804 & 27 & 60 & 0.639604 \\
\hline & 12000 & 27 & 60 & 1.341609 & 27 & 60 & 1.326009 & 27 & 60 & 1.326009 \\
\hline & 30000 & 27 & 60 & 3.354022 & 27 & 60 & 3.291621 & 28 & 62 & 3.416422 \\
\hline \multirow{4}{*}{ (49) } & 3000 & 41 & 86 & 3.276021 & 41 & 86 & 3.244821 & 42 & 88 & 3.322821 \\
\hline & 6000 & 40 & 84 & 10.670468 & 40 & 84 & 10.654868 & 41 & 86 & 10.90447 \\
\hline & 12000 & 40 & 84 & 37.48704 & 40 & 84 & 37.799042 & 42 & 88 & 39.655454 \\
\hline & 30000 & 42 & 88 & 250.459606 & 42 & 88 & 249.367599 & 44 & 92 & 261.020873 \\
\hline \multirow{4}{*}{$(50)$} & 3000 & 35 & 72 & 0.0624 & 35 & 72 & 0.0624 & 35 & 72 & 0.0468 \\
\hline & 6000 & 36 & 74 & 0.093601 & 36 & 74 & 0.109201 & 36 & 74 & 0.078 \\
\hline & 12000 & 38 & 78 & 0.171601 & 38 & 78 & 0.156001 & 38 & 78 & 0.156001 \\
\hline & 30000 & 39 & 80 & 0.452403 & 39 & 80 & 0.390002 & 39 & 80 & 0.421203 \\
\hline \multirow{3}{*}{ (51) } & 3000 & 28 & 97 & 1.918812 & 51 & 183 & 3.432022 & 51 & 183 & 3.447622 \\
\hline & 6000 & 47 & 164 & 10.608068 & 56 & 218 & 13.104084 & 56 & 218 & 13.088484 \\
\hline & 12000 & 42 & 135 & 30.872598 & 37 & 160 & 31.964605 & 37 & 160 & 31.886604 \\
\hline \multirow{3}{*}{ (52) } & 3000 & 569 & 1582 & 193.176038 & 800 & 2260 & 272.783349 & 800 & 2260 & 272.627348 \\
\hline & 6000 & 800 & 2184 & 1074.254086 & 800 & 2200 & 1077.140105 & 800 & 2200 & 1074.83129 \\
\hline & 30000 & 728 & 2025 & 24653.74284 & 784 & 2183 & 26511.62115 & 784 & 2183 & 26511.71475 \\
\hline
\end{tabular}




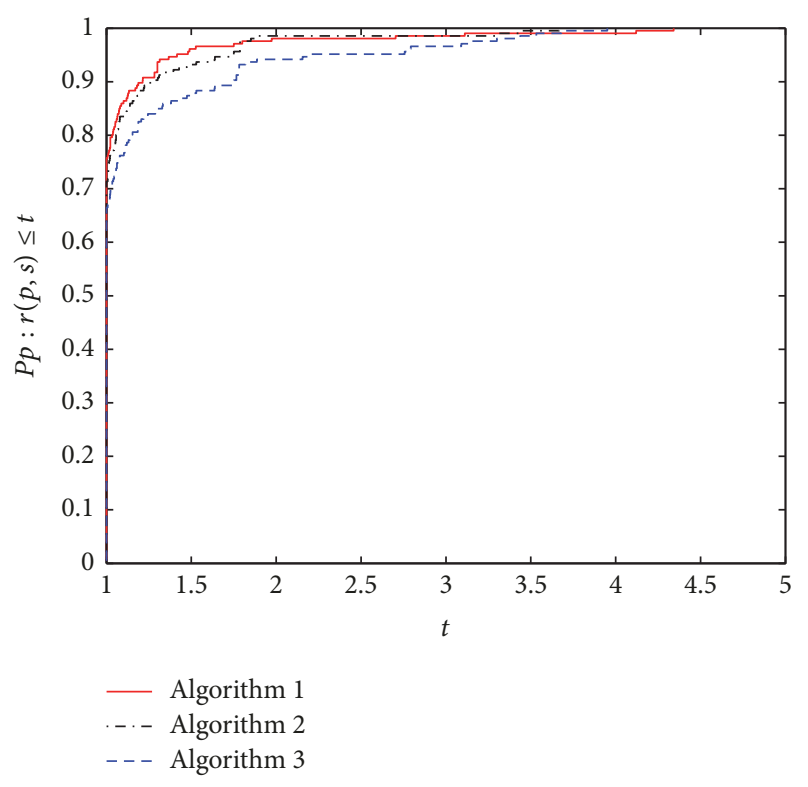

FIGURE 2: NFG performance of these methods.

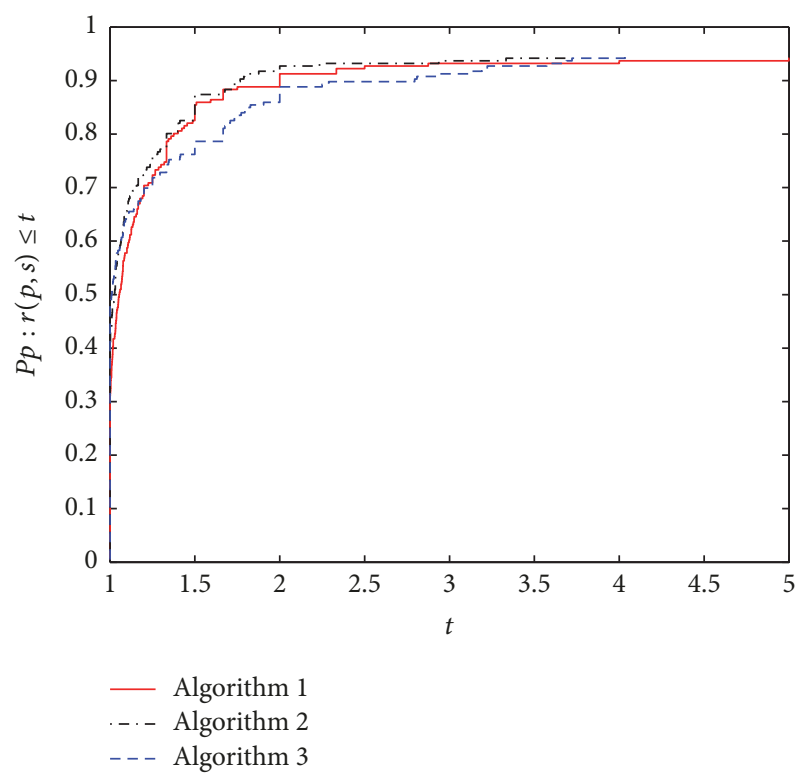

FIGURE 3: CPU time performance of these methods.

\section{Conclusions}

This paper proposes a modified HS three-term conjugate gradient algorithm for large-scale optimization problems and the given algorithm has some good features.

(1) The modified HS three-term conjugate gradient possesses a trust region property, which makes the global convergence of the general functions easy to get. However, the normal HS formula including many other conjugate gradient formulas does not have this feature, which may be the crucial point for the global convergence of the general functions.

(2) The largest dimension of the test problems is 30000 variables and the numerical results show that the presented algorithm is competitive to other similar methods. More experiments will be done to prove the performance of the proposed algorithm in the future.

\section{Conflicts of Interest}

The authors declare that there are no conflicts of interest regarding the publication of this paper.

\section{Authors' Contributions}

Mr. Xiangrong Li and Dr. Songhua Wang wrote this paper in English. Dr. Zhongzhou Jin and Dr. Hongtruong Pham carried out the experiment. All the authors read and approved the final manuscript.

\section{Acknowledgments}

This work is supported by the National Natural Science Foundation of China (Grants nos. 11661009 and 11261006), the Guangxi Science Fund for Distinguished Young Scholars (no. 2015GXNSFGA139001), the Guangxi Natural Science Key Fund (no. 2017GXNSFDA198046), and the Basic Ability Promotion Project of Guangxi Young and Middle-Aged Teachers (no. 2017KY0019).

\section{References}

[1] G. Yuan and Z. Wei, "Convergence analysis of a modified BFGS method on convex minimizations," Computational optimization and applications, vol. 47, no. 2, pp. 237-255, 2010.

[2] R. Fletcher and C. M. Reeves, "Function minimization by conjugate gradients," The Computer Journal, vol. 7, pp. 149-154, 1964.

[3] M. R. Hestenes and E. Stiefel, "Methods of conjugate gradients for solving linear systems," Journal of Research of the National Bureau of Standards, vol. 49, pp. 409-436 (1953), 1952.

[4] E. Polak and G. Ribière, "Note sur la convergence de méthodes de directions conjuguées," Revue Française d'Informatique et de Recherche Opérationnelle, vol. 3, no. 16, pp. 35-43, 1969.

[5] B. T. Polyak, "The conjugate gradient method in extremal problems," USSR Computational Mathematics and Mathematical Physics, vol. 9, no. 4, pp. 94-112, 1969.

[6] G. Yuan, Z. Meng, and Y. Li, "A modified Hestenes and Stiefel conjugate gradient algorithm for large-scale nonsmooth minimizations and nonlinear equations," Journal of Optimization Theory and Applications, vol. 168, no. 1, pp. 129-152, 2016.

[7] G. Yuan and M. Zhang, "A three-terms Polak-Ribière-Polyak conjugate gradient algorithm for large-scale nonlinear equations," Journal of Computational and Applied Mathematics, vol. 286, pp. 186-195, 2015.

[8] L. Zhang, W. Zhou, and D.-H. Li, "A descent modified PolakRibière-Polyak conjugate method and its global convergence," IMA Journal of Numerical Analysis (IMAJNA), vol. 26, no. 4, pp. 629-640, 2006.

[9] G. Yuan, Z. Wei, and X. Lu, "Global convergence of BFGS and PRP methods under a modified weak Wolfe-Powell line search," Applied Mathematical Modelling: Simulation and Computation for Engineering and Environmental Systems, vol. 47, pp. 811-825, 2017. 
[10] A. S. Nemirovsky and D. B. Yudin, Problem complexity and method efficiency in optimization, John Wiley \& Sons, Inc., NY, New York, USA, 1983.

[11] G. Yuan, Z. Sheng, B. Wang, W. Hu, and C. Li, "The global convergence of a modified BFGS method for nonconvex functions," Journal of Computational and Applied Mathematics, vol. 327, pp. 274-294, 2018.

[12] N. Andrei, "An unconstrained optimization test functions collection," Advanced Modeling and Optimization, vol. 10, no. 1, pp. 147-161, 2008.

[13] Y. Wu, "A modified three-term PRP conjugate gradient algorithm for optimization models," Journal of Inequalities and Applications, Paper No. 97, 14 pages, 2017.

[14] E. D. Dolan and J. J. Moré, "Benchmarking optimization software with performance profiles," Mathematical Programming, vol. 91, no. 2, pp. 201-213, 2002. 


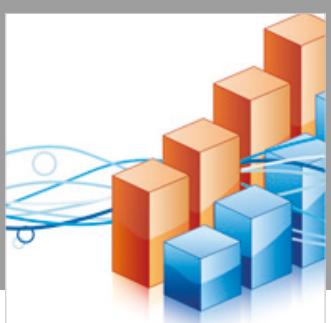

Advances in

Operations Research

\section{-n-m}
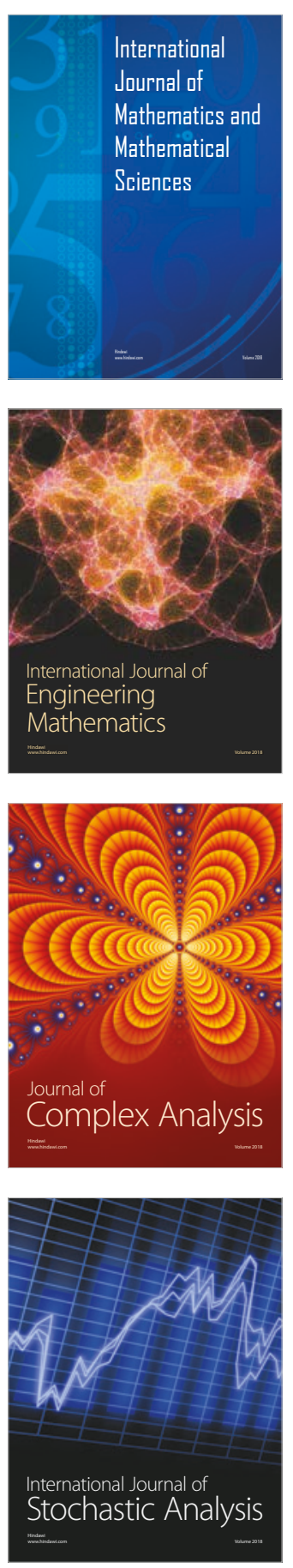
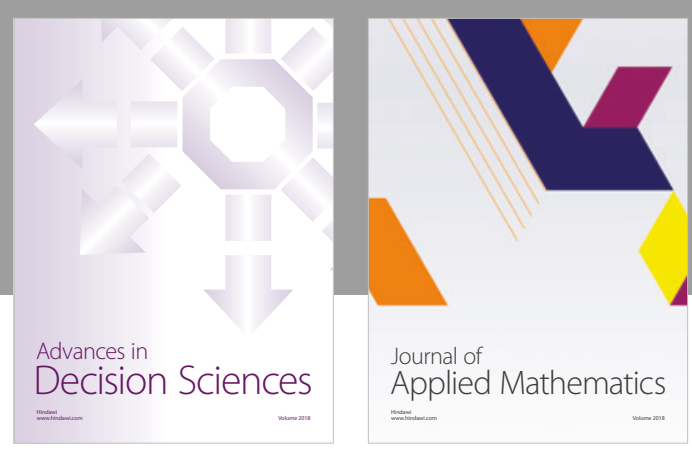

Journal of

Applied Mathematics
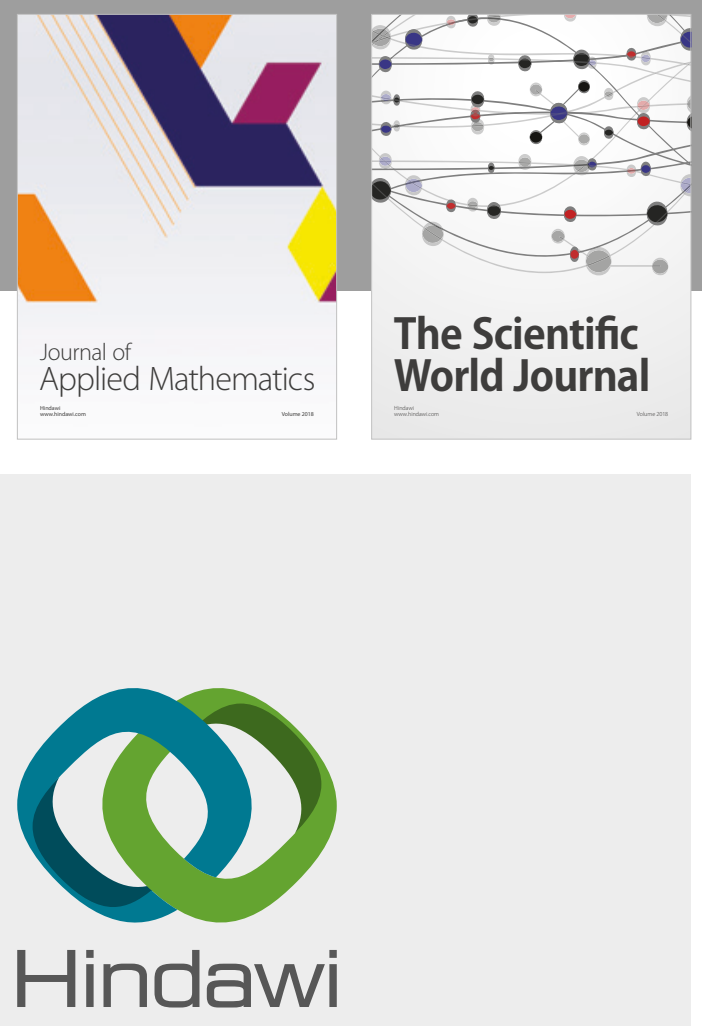

Submit your manuscripts at

www.hindawi.com

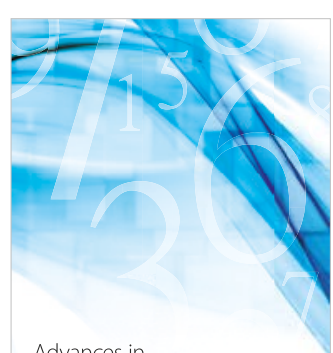

Advances in
Numerical Analysis
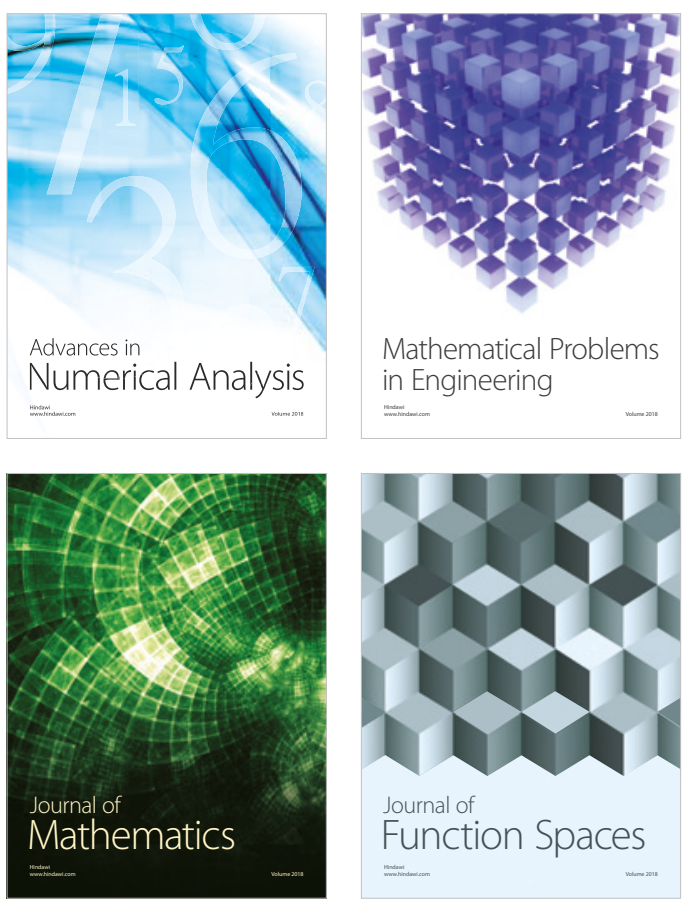

Mathematical Problems in Engineering

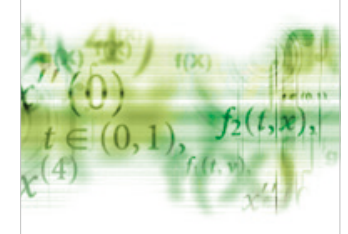

International Journal of

Differential Equations

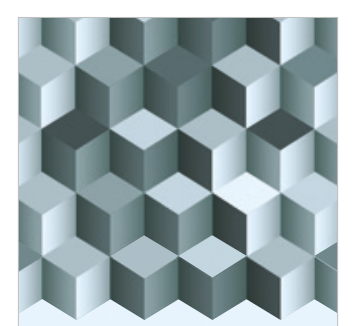

Journal of

Function Spaces

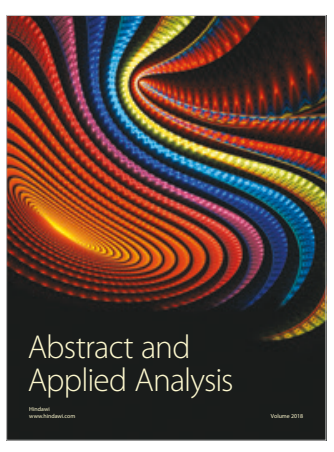

The Scientific

World Journal

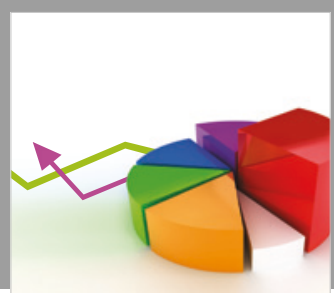

Journal of

Probability and Statistics
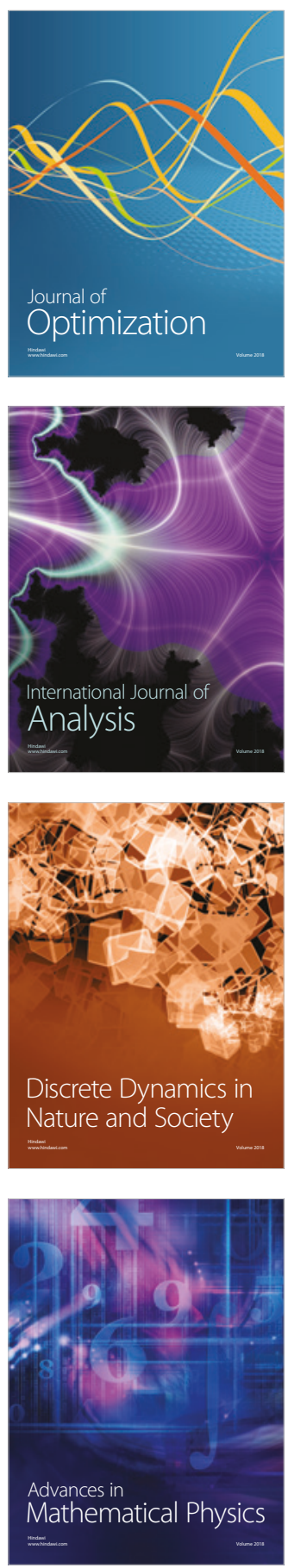